\title{
Practical Routing Protocol Models to Improve Network Performance and Adequacy
}

\author{
Mohammad Alhihi \\ Philadelphia University, Amman, Jordan \\ Email:Malhihi@philadelphia.edu.jo, alhihimohammad25@gmail.com
}

How to cite this paper: Alhihi, M. (2017) Practical Routing Protocol Models to Improve Network Performance and Adequacy. Journal of Computer and Communications, 5, 114-124.

https://doi.org/10.4236/jcc.2017.56007

Received: March 10, 2017

Accepted: April 25, 2017

Published: April 28, 2017

Copyright $\odot 2017$ by author and Scientific Research Publishing Inc. This work is licensed under the Creative Commons Attribution International License (CC BY 4.0).

http://creativecommons.org/licenses/by/4.0/ (c) (i) Open Access

\begin{abstract}
The telecommunications network has become an essential facility of our daily life, which is the reason why a good number of the recent researches are directed to it. In this paper, the performance of routing protocols for selected network and the optimum way of utilizing the network resources is proposed, through improving the routing method protocols to obtain the most accurate communication models, which give the best network performance, taking into consideration that the protocol time interval to recalculate the future routing step is agreed to be our performance criteria, which is then used to determine the total amount of incoming traffic normalized at the best network performance with respect to the network capacity as well. Moreover, adaptive routing evaluation methods have been mathematically investigated in this work for telecommunication networks (TN), more precisely, developing the infrared radiation (IR) routing methods for hybrid $\mathrm{TN}$ applications, in terms of implementation conditions at a certain guaranteed quality of service, where we give a particular interest in analyzing routing methods based on the fact that the distribution information is contained in the routing variables, rather than in the state variables. The protocol that deals with the unbalanced traffic distribution over TN nodes has been investigated in this paper as well, and simulation results show good progress in the proposed protocol over the traditional protocols.
\end{abstract}

\section{Keywords}

Telecommunications Network, Routing Protocols, Hybrid Network

\section{Introduction}

Telecommunication network (NT) routing protocols are such an old topic that controls the network performance, which is discussed in many old and recent research work, for example, in 1998 [1], deferent multi-hop wireless ad hoc 
network routing protocols are compared for different scenarios to investigate the best protocol that gives the best network performance. This good work was directly followed with such important work deals with the same field in [2], where the needed dynamic routing protocols were investigated and the performance compression in mobile network between dynamic source routing and ad hoc on-demand distant vector routing was provided as well.

In [3] the protocols of attacking and defending the network were proposed to show how important to choose the routing protocol in ad hoc network.

Most recent work is aimed at power efficient protocols for different applications, such as wireless sensor network [4] [5], and [6] proposed novel protocols for wireless networks resulted in good power saving and better automatic repeat request (ARQ) through improving the routing mechanism. In [7], mobile network routing protocol was introduced and it shows how the routing protocols do improve the power and bandwidth efficiently even for such small networks as a result of improving the PER.

Routing protocols is becoming more and more updated research work because its ability to adapt with other techniques, such as cooperative network and network coding network [7] and [8].

The rest of the paper is organized as follows: Section 2 deals with the experimental study of the developed models and routing methods in TN, which shows how the proposed protocol works in different scenarios, hence Section 2 is divided to two sub-sections, which are 2.1 which shows the Block diagram of the experimental model and 2.2 which shows how to check the adequacy of the developed routing models. Section 3 gives the conclusion for the paper.

In this paper, the performance of routing protocols for selected network and the optimum way of utilizing the network resources is proposed, unlike [9] where an exact algorithm based on the Branch and Bound principle is proposed and then improved by the Lookahead technique.

Both [10] and [11] propose sensor network for underwater application, where in [10] the network architecture for underwater acoustic sensor networks was shown, over different layers such as MAC and routing protocols for the application layer over the acoustic channel, and in [11] depth division-policy was applied to obtain an efficient and scalable version of depth-based routing protocol to reduce the consumed energy and the delay in communication from end-toend, for underwater sensor network.

In [12] the multipath routing in layer 2 networks was investigated using a non-shortest-path routing approach, equal preference multipath that can generate more paths than equal cost multipath.

Network performance is identified as the property of TN to provide a given information transmission probability from a source to a destination with maintaining the quality of service transmission [13].

Moreover, in this paper, the protocol time interval to recalculate the future routing step is agreed to be our performance criteria, and hence the obtained results are based on this. In general, the value of the network performance de- 
pends on the network configuration, bandwidth, the network atmosphere, and the network variables as shown in Equation (1):

$$
P(k)=\sum_{i=1}^{N} \sum_{j=1}^{N} c_{i, j}(k) u_{i, j}^{j}(k), \text { where } i \neq j .
$$

where $P(k)$ is the network performance, $u_{i, l}^{j}(k)$ is the routing variables taking into consideration that communication input stream availability is assumed, otherwise, the routing variables are zeroes, and $C_{i, l}^{j}(k)$ is the network bandwidth.

Relative performance of the network at the time interval can be written as

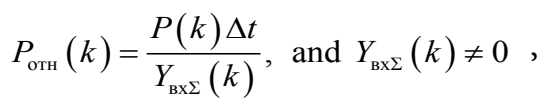

where $Y_{\mathrm{B} \times \Sigma}(k)$ is the total load value supplied to the network at $M$ time interval and it is:

$$
Y_{\mathrm{B} \Sigma}(k)=\sum_{i=1}^{N} \sum_{j=1}^{N} y_{\mathrm{Bx} i, j}(k), i \neq j,
$$

where $y_{\mathrm{B} x i, j}(k)$ is the volume load entering the node $V_{i}$ and intended site $V_{j}$. Unlike $y_{i, j}(k)$ which indicates the real amounts of data, rather than the predicted value.

In order to further comparative analysis of simulation results for different network configurations, we introduce network performance $P_{\mathrm{H}}(k)$ and the total amount of incoming traffic $Y_{\text {вхн }}(k)$ normalized with respect to the maximum possible in the network performance values, that is, with respect to its capacity:

$$
P_{\mathrm{H}}(k)=\frac{P(k)}{C(k)} 100 \%
$$

and

$$
Y_{\mathrm{BX \textrm {H }}}(k)=\frac{Y_{\mathrm{BX \Sigma}}(k)}{C(k) \Delta t} 100 \%
$$

where $C(k)$ - is the bandwidth at $k-M$ time interval. Normalized performance during simulation $T_{\mathrm{M}}$, consisting of $v$ at the time slots $T_{\mathrm{M}}=v \Delta t$ are defined as follows:

$$
P_{\text {н }}=\frac{\sum_{k=1}^{v} P(k)}{\sum_{k=1}^{v} C(k)} 100 \% .
$$

where $P_{\mathrm{H}}$ is the normalized performance. Similarly, the normalized load volume entering the network during the simulation $T_{\mathrm{M}}=v \Delta t$

$$
Y_{\mathrm{BX \textrm {H }}}=\frac{\sum_{k=1}^{v} Y_{\mathrm{BX} \Sigma}(k)}{\Delta t \sum_{k=1}^{v} C(k)} 100 \% .
$$


So, as network performance can be regarded as an indicator for firstly, the resources efficiency usage represented by the network utilization capacity of the nodes buffer memory, and secondly, the paths transmission bandwidth. The first indicator is expressed in terms of the state variables, and the second indicator is expressed through the route variables. The node $V_{i}$ utilization rate capacity of the buffer memory $R_{i}$ can be defined as:

$$
R_{i}=\frac{1}{x_{i}^{\max }}\left(\sum_{j=1}^{N} x_{i, j}(k)\right), j \neq i
$$

where $x_{i}^{\max }$ is the maximum allowable amount of information that can be stored in a buffer node device $V_{i}$ and $x_{i, j}(k)$ is the data volume which represents the network information.

However, given the particular interest in analyzing routing methods to the way in which there is a distribution of information flows and the fact that the information contained in the routing variables, rather than the state variables, special importance attaches to indicators that are based on these variables: bandwidth utilization of a separate tract transmission $E_{i, j}$ lots of traffic passed through it $K_{i, j}(k)$ and channel utilization of network resources on the whole $K_{\mathrm{ECP}}(k)$ on $k-M$ time interval as it can be summarized in [13]:

$$
\begin{gathered}
K_{i, j}(k)=\sum_{m=1}^{N} u_{i, j}^{m}(k), m \neq i, \\
K_{\mathrm{ECP}}(k)=\frac{\sum_{i=1}^{N} \sum_{j=1}^{N} K_{i, j}(k)}{N_{\text {тр }}}, i \neq j,
\end{gathered}
$$

where $N_{\text {тр }}$ is the number of transmission paths in a full mesh network where $N_{\text {тр }}=N(N-1)$. According to Equations (8) and (10) the parameters reflecting the degree of utilization of the channel resources can be obtained directly on the basis of the vectors $U(k)$ calculated based on the physical meaning of their components. Opportunity evaluation in the models of utilization of network resources is particularly important in the practice routing methods which is based on the shortest path algorithms.

As the main problem in such protocols, is the inefficiency resulted from the unbalanced distribution of traffic over nodes or over transmission time intervals.

In this regard, the introduction of performance $K_{i, j} E$ and $K_{\mathrm{ECP}}$ opens the possibilities for the evaluation and comparative analysis methods to develop an active protocol that can deal with this problem.

The proposed protocol deals with the problem of unbalanced distribution of the traffic over the nodes. The practical implementation of the introduced shortest path model satisfies the condition of Equation (11) [14]

$$
K_{i, j}(k) \leq K_{\max } \leq 1,
$$

which provides a reduction in the degree of channel resources utilization, communication routes that are part of the shortest paths, and encourages the use of bypass routes resulting to mitigating the effect of the congestion in the networks. 
The value $K_{\max }$ in such setting is determined by the configuration and the particular network traffic selected for each network separately.

\section{Experimental Study of the Developed Models and Routing Methods in TN}

\subsection{Block Diagram of the Experimental Model}

The proposed work in this paper is based on experimental study of the developed models, where the routing methods have been carried out by means of mathematical modeling and simulation. The degree of the modeling process detailed information that exchanges in the TN, is obtained by the experimental method as well, which directly reflects the content of the developed models and routing methods, i.e., collecting and processing information on the state of the network, calculation and implementation of the routing tables, ..., etc. A number of issues such as queuing mechanisms, and mechanisms for restricting traffic alignment were not considered because their effect is assumed to be the same for all traditional and proposed protocols.

The block diagram of the experiment is shown in Figure 1. The central unit (block 4) is the unit for forming the routing tables, which its operation is based on the algorithm proposed models and routing methods. The optimization interval $\left(t_{0}, t_{k}\right)$ which is proposed to solve the routing problem is changing from $t_{0}=t_{h}$ to $t_{\kappa}=t_{h}+T$ where $T=a \Delta t$ is the projection period, and $h$ is the current number of time interval $\Delta t$ or the modeling phase. The total simulation

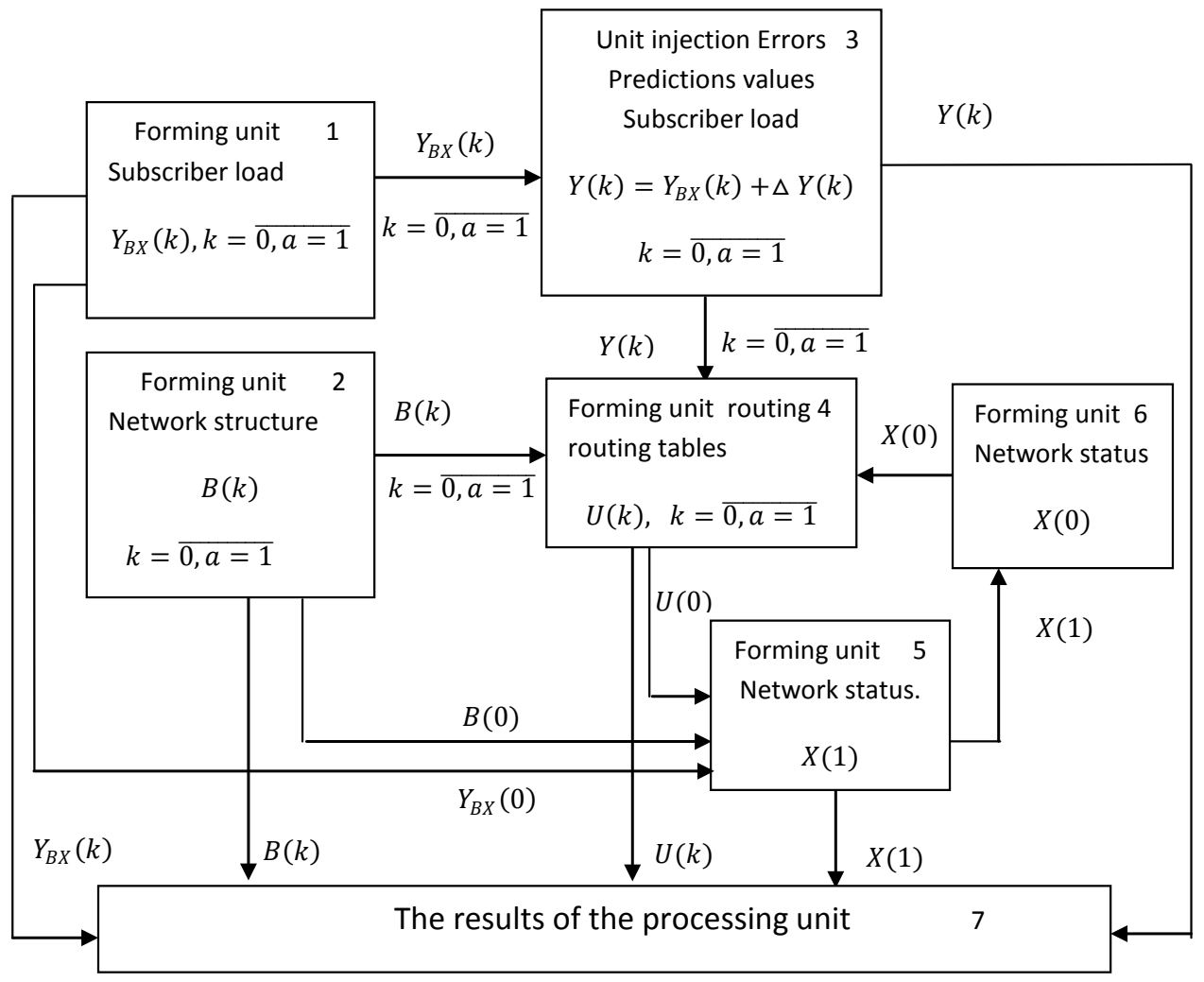

Figure 1. Block diagram of the experimental model. 
time is $T_{\mathrm{M}}=v \Delta t$ as realized by routing the prediction model, has the property that generates the set of the routing tables as shown in Figure 1.

Initial data for constructing information $\mathrm{MT}$ is entering the current blocks 2, 3 , 5, and 6 Figure 1, forming the current status of the network, which is expressed as a matrix and the initial value $B(k)$ loading buffer queues at the nodes $X(0)$, and on the projected value of the incoming load $Y(k)$, where the predicted accuracy is governed by unit 3 . In general, the prediction error values are determined by the incoming flow, in which the prediction is realized. However, in our proposed patterns study and routing techniques; it is assumed that $Y_{\mathrm{Bx}}(k)$ dynamic load changes is known with some specified error $\Delta Y(k)$, due to the deterministic nature of the proposed routing patterns.

Though the process proceeds to the network load is random, it allocates a separate task of synthesis vectors $Y_{\mathrm{в}}(k)$. During the simulation of the routing process it assumes that the volume entering the network load is a function of time and it is a random process with parameters

$M\left[y_{\mathrm{Bx} i, j}(k)\right]=y_{\mathrm{Bx} \mathrm{cp} i, j}(k)$ and $D\left[y_{\mathrm{Bx} i, j}(k)\right]=\sigma_{y i, j}^{2}(k)$.

As the study that [15] [16] showed the statistical characteristics of the telecommunications traffic; the volume of the load entering the network is a random variable whose distribution law in most cases can be approximated by the normal or the log-normal law. Taking into consideration that for the proposal of Gaussian model traffic description applicability in TN, a large number of independent sources of active (up to several thousand) are required as specified in [16]. In the absence of a specified number of sources per hour or lower activity of the amount of traffic on the network; an approximated lognormal Gaussian model traffic distribution is proposed in [16].

The requirement for a high number of active sources is due to the central limit theorem: The law of the probability distribution of the independent random variables summation with the same order of unlimited, increases in terms of independence from the laws of distribution tends to a normal distribution. On the practical side prerequisite to the use of this theorem, it appears that the level of wide area network (WAN) streams arrive at the router from aggregating multiple nodes, each of which its density reaches up to 10,000 subscriber terminals [17]. Also it is important to notice that in multiservice network streams, entering the network by subscribers are heterogeneous (data, voice, video on demand, video conferencing), among which highlight the difficult prevailing traffic type. As a result, it can be regarded as distributed according to the law, which is close to normal. We are talking about a truncated normal law, which is due to the physical meaning of the variables $y_{\mathrm{B} x i, j}(k)$ where $y_{\mathrm{Bx} i, j}(k)>0$.

During the experimental studies of the proposed models and routing methods by means of mathematical modeling; the simulation method is used as the input load datasets with the two distribution laws. Thus, in the simulation process of information exchange in the TN; the collected resulting data in this work is for:

- Network structure and capacities of transmission paths on the basis of which the matrix is formed $B(k)$. 
- Boot buffer queues at the network nodes $X(0)$.

- The value of the incoming user traffic $Y_{\mathrm{BX}}(k)$ and the accuracy of its prediction $\Delta Y(k)$.

- Prediction interval and the interval $T$ recalculation $\Delta t$.

- The total time of simulation $T_{\mathrm{M}}$ and size $v$.

In the modeling process at each stage, it is possible to monitor and assess the state of the network $X(h)$, utilization of channel resources of $K_{i, j}(h)$ and $K_{\mathrm{ECP}}(h)$, and the introduction of an effective performance, in terms of network performance for its relative and normalized values.

\subsection{Check the Adequacy of the Developed Routing Models}

The purpose of checking the adequacy design for mathematical routing methods for NT models is to determine their correctness and, ultimately, the possibility of (borders) application.

In the absence of the possibility of direct implementation of the proposed research models and methods for routing in the existing $\mathrm{TN}$, and obtaining experimental data to support or disprove their validity, we consider a number of key factors by which to judge the adequacy of the proposed models and methods.

The adequacy of the model and the accuracy obtained with the help of the results provided by:

- Using modern and proven mathematical apparatus: the theory of differential-difference equations, systems theory and optimal control theory.

- Sound (correct) the selection criteria and optimal performance.

- The large number of factors is considered, which is expressed in the form of restrictions, each of which is grounded and has a clear physical meaning.

Simulation of the proposed routing method performed for different network structures, however, in this case, as shown by the results of the simulation, the maximum efficiency of the proposed methods based on models in state-space emerged in the implementation of irregular network structures, which are often used in practice. By irregular network structures include structures in which the vertices have different degrees, as opposed to regular, and fully connected.

The most typical representative of the irregular structure is the so-called "fish" as shown in Figure 2, [16] [18].

A distinguishing feature of "fish" is the presence of two independent paths between a pair of nodes in Figure 2, on nodes 1 and 5, which are of different

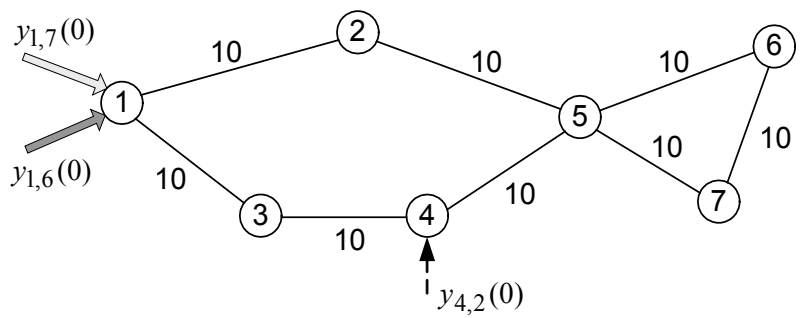

Figure 2. Example for sub-optimal distribution network protocol (fish). 
lengths. Specificity classical IP-routing leads to that all flows from node 1 to node 5 will be directed along the shortest path (through node 2), while the path length is a little higher, it will not be involved at all. This example clearly illustrates the disadvantages routing along the shortest path, which, as a challenge to the individual quality indicators as the objective function, leads to suboptimal across the distribution network flows.

Consider the detailed work on the network model example as shown in Figure 2, which will demonstrate the essence of the proposed solutions for the routing problems, resulting to efficiency improvement for the network functions.

Assuming that the network receives information on a service flow with three projected intensity received $\zeta_{1,6}(0)=6, \zeta_{1,7}(0)=6, \zeta_{4,2}(0)=3 \mathrm{Mbps}$. For the propose of simplify; consider a model without the option of prediction choosing the simulation time at $T_{\mathrm{M}}=\Delta t=10 \mathrm{~s}$.

Based on above, the amount of data entering the network comprise $y_{1,6}(0)=60, y_{1,7}(0)=60, y_{4,2}(0)=30$ Mbps. Assuming that the network does not boot, i.e., $X(0)=0$.

In organizing the routing process according to the shortest path algorithms path 1-2-5-6 will be used for transmission of said stream, 1-2-5-7 and 4-5-2 as shown in Figure 3.

Given that the path from node 1 to the nodes 6 and 7 have a common region whose bandwidth is $10 \mathrm{Mbps}$, for a time $\Delta t$ streams $y_{1,6}(0)$ and $y_{1,7}(0)$ fully serviced and are in turn the end of the simulation $x_{1,6}(1)$ and $x_{1,7}(1)$ take the value of 10 Mbps.

Application of the developed routing method will provide targeted functionality, with the result of the decision is a vector routing variables are shown in $\mathrm{Ta}$ ble 1, which corresponds to the distribution of flows shown in Figure 4.

The model generates a distribution of network resources that the entire amount of data received by the network, will be serviced, and the service information is

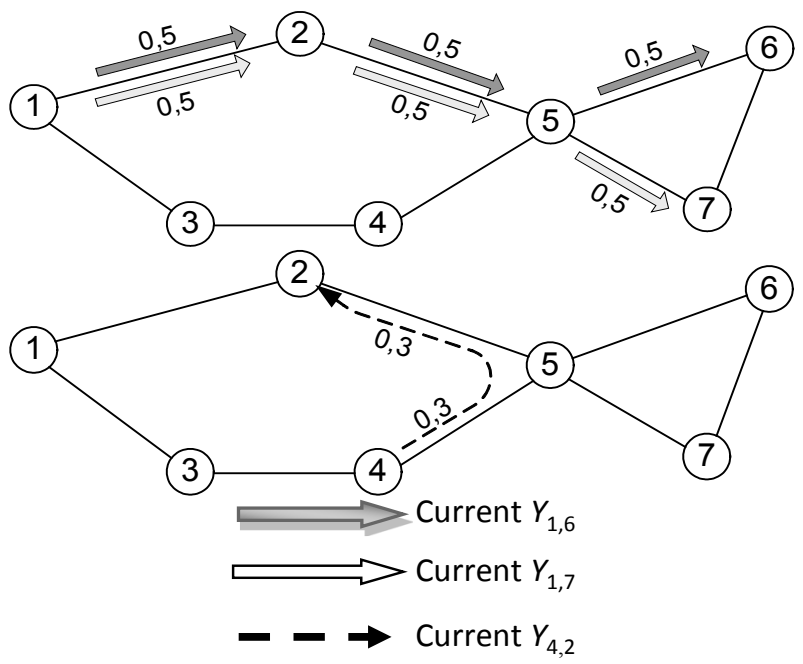

Figure 3. The distribution of flows in the network according to a shortest path algorithm. 
Table 1. The results of solving the routing problem.

\begin{tabular}{llllllll}
\hline$i$ & $j$ & $m$ & $u_{i, j}^{m}(0)$ & $i$ & $j$ & $m$ & $u_{i, j}^{m}(0)$ \\
\hline 1 & 2 & 2 & 0.12 & 3 & 4 & 7 & 0.24 \\
1 & 2 & 6 & 0.36 & 4 & 3 & 2 & 0.12 \\
1 & 3 & 6 & 0.24 & 4 & 5 & 2 & 0.18 \\
1 & 2 & 7 & 0.36 & 4 & 5 & 6 & 0.24 \\
1 & 3 & 7 & 0.24 & 4 & 5 & 7 & 0.24 \\
2 & 5 & 6 & 0.36 & 5 & 2 & 2 & 0.18 \\
2 & 5 & 7 & 0.36 & 5 & 6 & 6 & 0.6 \\
3 & 1 & 2 & 0.12 & 5 & 7 & 7 & 0.6 \\
3 & 4 & 6 & 0.24 & - & - & - & - \\
\hline
\end{tabular}



(a)

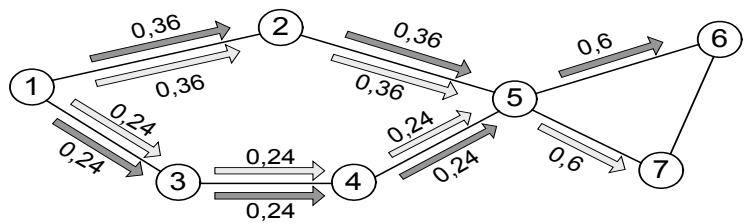

(b)

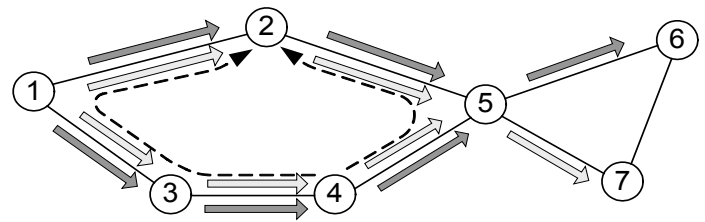

(c)

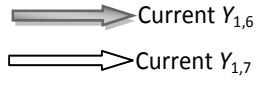

- - $\rightarrow$ Current $Y_{4,2}$

Figure 4. Flow distribution in accordance with the proposed model routing.

not the same path, but in several simultaneously. As a result, unlike the routing algorithm by the shortest path, there is no accumulation of data in one of the nodes that increases network capacity and prevents overload eventually. This example illustrates the fact that when using the routing of the proposed model is achieved by a balanced use of resources, that is the most uniform loading units and transmission paths.

\section{Conclusions}

In this work, our practical study introduces the performance of routing protocols for selected network and the optimum way of utilizing the network re- 
sources. The achieved performance has been obtained by the proposed routing method protocols that determines the most accurate communication models and hence estimates the expected network best performance.

The protocol time interval was used in this paper to recalculate the future routing step and considers it to be our performance criteria.

The proposed protocol shows how the network capacity improves when choosing the less traffic path (shortest time interval) rather than the shortest one.

The block diagram for the proposed protocol is provided with the proposed check routing model adapted in this work with practical calculations that showed the optimum path between two suggested nodes based on the less traffic path resulting to valuable data rate saving in the whole network.

Finally, the paper shows that the proposed protocols deal with the unbalanced traffic distribution over TN nodes and how good progress this protocol achieves in such scenario.

The future work will be directed to more specific protocols that practically improves the performance of specific applications such as condensed mobile network, intensive wireless sensor network, and other applications such as [19].

\section{Acknowledgements}

The authors of this paper would like to thank the Philadelphia University for their support and for assuring such good researching atmosphere for us. Moreover, authors truly would like to thank the reviewers of this paper for the good advice they provided which improved this work significantly.

\section{References}

[1] Broch, J., Maltz, D.A., Johnson, D.B., Hu, Y.-C. and Jetcheva, J. (1998) A Performance Comparison of Multi-Hop Wireless Ad Hoc Network Routing Protocols. Proceedings of the 4th Annual ACM/IEEE International Conference on Mobile Computing and Networking, Dallas, Texas, 25-30 October 1998, 85-97.

[2] Perkins, C.E., Royer, E.M., Das, S.R. and Marina, M.K. (2001) Performance Comparison of Two On-Demand Routing Protocols for Ad Hoc Networks. IEEE Personal Communications, 8, 16-28. https://doi.org/10.1109/98.904895

[3] Hu, Y.-C., Perrig, A. and Johnoson, D.B. (2003) Rushing Attacks and Defense in Wireless Ad Hoc Network Routing Protocols. Proceedings of the 2nd ACM Workshop on Wireless Security, San Diego, 19 September, 2003, 30-40.

https://doi.org/10.1145/941311.941317

[4] Attar, H., Stankovic, L. and Stankovic, V. (2012) Cooperative Network-Coding System for Wireless Sensor Networks. IET Communications, 6, 344-352.

https://doi.org/10.1049/iet-com.2011.0143

[5] Attar, H., Vukobratovic, D., Stankovic, L. and Stankovic, V. (2011) Performance Analysis of Node Cooperation with Network Coding in Wireless Sensor Networks. 4th IFIP International Conference on New Technologies, Mobility and Security, Paris, 7-10 February 2011, 1-4. https://doi.org/10.1109/NTMS.2011.5721048

[6] Alhihi, M. (2017) Network Coding for Wireless Sensor Network Cluster over Rayleigh Fading Channel: Finite State Markov Chain. International Journal of Commu- 
nications, Network and System Sciences, 10, 1-11.

[7] Attar, H., Stankovic, L., Alhihi, M. and Ameen, A. (2014) Deterministic Network Coding over Long Term Evaluation Advance Communication System. 4th International Conference on Digital Information and Communication Technology and Its Applications (DICTAP), Bangkok, 6-8 May 2014, 56-61. https://doi.org/10.1109/dictap.2014.6821657

[8] El-Hihi, M., Attar, H., Solyman, A.A.A. and Stankovic, L. (2016) Network Coding Cooperation Performance Analysis in Wireless Network over a Lossy Channel, M Users and a Destination Scenario. Communications and Network, 8, 257-280. https://doi.org/10.4236/cn.2016.84023

[9] Molnar, M. (2016) Exact Algorithm to Solve the Minimum Cost Multi-Constrained Multicast Routing Problem. Journal of Computer and Communications, 4, 57-79. https://doi.org/10.4236/jcc.2016.414005

[10] Khosravi, M.R., Salari, R. and Rostami, H. (2016) A Solution for Scalable Routing in Depth Divisions-Based DUSNs via Adding a Scalable Parameter to Control Depth Clusters: Creating an Energy Efficient and Low Delay NI-Independent Communication Protocol. Journal of Computer and Communications, 4, 55-61. https://doi.org/10.4236/jcc.2016.47008

[11] Sharif-Yazd, M., Khosrav, M.R. and Moghimi, M.K. (2017) A Survey on Underwater Acoustic Sensor Networks: Perspectives on Protocol Design for Signaling, MAC and Routing. Journal of Computer and Communications (JCC), 5, 12-23.

[12] Hou, T.-C. and Tsai, H.-C. (2016) Equal Preference Multi-Path Routing for L2 Hierarchical Networks. Journal of Computer and Communications, 4, 37-56. https://doi.org/10.4236/jcc.2016.414004

[13] Evseev, O. (2002) Experimental Research of Dynamic Model of Adaptive Datagram Routing in Telecommunications Networks. Radiotekhn Single: Vseukr. Interdepartmental. Scientific and Engineering. Sat, 133, 20-26.

[14] Olifer, V.G. and Olifer, N.A. (2001) Art Traffic Optimization. Journal of Set-O LAN Solutions, No. 12, 38-47.

[15] Norros, I. (1995) The Management of Large Flows of Connectionless Traffic on the Basis of Self-Similar Modeling. IEEE International Conference on Communications, ICC95 Seattle, “Gateway to Globalization”, Seattle, 18-22 June 1995, 451-455. https://doi.org/10.1109/ICC.1995.525210

[16] Norros, I. and Pruthi, P. (1996) On the Applicability of Gaussian Traffic Models. Proceedings of the 13 th Nordic Teletraffic Seminar, Trondheim, 37-50.

[17] Gurgenidze, A.T. and Koresh, V.I. (2003) Multiservice Networks and Broadband Services. Science and Engineering, 393-400.

[18] Wang, J. (2002) Acquires IP Routing Intelligence. Journaltevyh se-LAN solutions, No. 6, 43-49.

[19] Attar, H. (2017) Data Combination over Physical Layer Using Network Coding with PUM Turbo Codes. Journal of Computer and Communications, 5, 32. 
Submit or recommend next manuscript to SCIRP and we will provide best service for you:

Accepting pre-submission inquiries through Email, Facebook, LinkedIn, Twitter, etc. A wide selection of journals (inclusive of 9 subjects, more than 200 journals)

Providing 24-hour high-quality service

User-friendly online submission system

Fair and swift peer-review system

Efficient typesetting and proofreading procedure

Display of the result of downloads and visits, as well as the number of cited articles Maximum dissemination of your research work

Submit your manuscript at: http://papersubmission.scirp.org/

Or contact jec@scirp.org 